\title{
Altered splicing associated with the pathology of inflammatory bowel disease
}

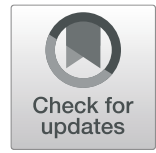

Kiera Berger ${ }^{1} \mathbb{D}$, Hari Somineni ${ }^{2,3}$, Jarod Prince ${ }^{2}$, Subra Kugathasan ${ }^{2}$ and Greg Gibson ${ }^{{ }^{*}}$

\begin{abstract}
Background: Aberrant splicing of individual genes is a well-known mechanism promoting pathology for a wide range of conditions, but disease is less commonly attributed to global disruption of exon usage. To explore the possible association of aberrant splicing with inflammatory bowel disease, we developed a pipeline for quantifying transcript abundance and exon inclusion transcriptome-wide and applied it to a dataset of ileal and rectal biopsies, both obtained in duplicate from 34 pediatric or young adult cases of ulcerative colitis and Crohn's disease.

Results: Expression and splicing covary to some extent, and eight individuals exhibited aberrant profiles that can be explained by altered ratios of epithelial to stromal and immune cells. Ancestry-related biases in alternative splicing accounting for $5 \%$ of the variance were also observed, in part also related to cell-type proportions. In addition, two individuals were identified who had 284 exons with significantly divergent percent spliced in exons, including in the established IBD risk gene CEACAM1, which caused their ileal samples to resemble the rectum.

Conclusions: These results imply that quantitative differences in splice usage contribute to the pathology of inflammatory bowel disease in a previously unrecognized manner.
\end{abstract}

Keywords: Inflammatory bowel disease, Splicing, RNA-seq, Percent spliced in, Gene expression

\section{Background}

Defective RNA splicing contributes to the etiology of a wide variety of diseases [1]. Single gene defects that weaken or abolish splice sites or activate cryptic ones have been associated with over 200 human diseases, including progeria, cystic fibrosis, muscular dystrophies, and some cancers [2-5]. Computational analyses have further identified variants in over 80,000 splicing regulatory motifs [6], and scores such as TraP (TRanscript inferred Pathogenicity Score) provide pre-computed predictions of likely splice defects for polymorphisms affecting all human genes [7]. Just as importantly, global mis-regulation of the splicing of hundreds of genes due to aberrant activity of components of the spliceosome, is known to contribute to pathology for a variety of conditions, notably myelodysplastic syndrome,

\footnotetext{
* Correspondence: greg.gibson@biology.gatech.edu

${ }^{1}$ School of Biological Sciences and Center for Integrative Genomics, Georgia Institute of Technology, Atlanta, GA 30332, USA

Full list of author information is available at the end of the article
}

myotonic dystrophy, several neurological disorders, and cancer metastasis [8-12].

The inflammatory bowel diseases (IBD), ulcerative colitis (UC), and Crohn's disease (CD) afflict approaching $1 \%$ of adults in developed countries and have been rising in prevalence globally for several decades [13]. They are well-known to involve aberrant gene expression in the gut $[14,15]$ as well as peripheral immune system $[16,17]$, and signatures of severe disease at diagnosis have been associated with progression to complicated disease or remission [18-20] and are being developed as biomarkers of therapeutic response [21]. There is also some indication that gene expression is to some extent ancestry-dependent, resulting in mis-regulation of pathways related to cytokine signaling, extracellular matrix function, and mitochondrial activity that is biased toward more adverse outcomes in African Americans [22]. To date, to our knowledge, there have not been any reports of splicing defects in IBD, so we asked whether transcriptome profiles assessed 
by the RNA-seq of bulk ileal and rectal tissues might provide evidence for unusual splice isoforms associated with IBD in a dataset of paired ileal and rectal biopsies from a cohort of 34 young individuals with $\mathrm{CD}$ or UC.

\section{Results}

Effects of disease, location, and ancestry on splicing and gene expression

In order to quantify the influences of disease, location, and ancestry on splicing and gene expression, we first computed the principal components (PC) for both the transcript abundance and PSI (percent spliced in) counts from the RNA-seq dataset and then generated a weighted sum of the influences on these measures. This principal component variance analysis revealed that three quarters $(75 \%)$ of the expression variability and one third $(33 \%)$ of the splicing variability was captured by the first ten principal components of the respective measures, indicating that gene level expression is far more variable than exon usage between rectal and ileal tissue. For gene expression, $40.3 \%$ of the variance was between locations (ileum and rectum), 2.3\% between ancestry groups (European and African), 0.2\% between disease subtypes (UC and $\mathrm{CD}$ ), and $0.8 \%$ captured by the interaction between location and disease. Corresponding percentages for the splicing variance were $20.7 \%$ between locations, $5.8 \%$ ancestry groups, $0.7 \%$ disease, and
$0.8 \%$ the interaction effect. These proportions and the contributions to each PC are provided in Table 1, which also shows that the variance contributions to PSI are relatively unaffected by the threshold of inclusion, being similar for datasets with 108,091 or 7001 exon bins. In both cases, then, consistent with previous studies, by far the largest effect is between ileum and rectum [23], a meaningful ancestry component is observed [22], and twice as much variance is due to the differences in the effect of disease on the two tissues than to disease across both tissues.

At the 5\% False Discovery Rate, there were 9569 differentially expressed genes by location, 1847 by ancestry, and just 556 by disease, although 1570 showed an interaction effect, implying that most disease effects, as expected, are specific to the ileum (in CD) or rectum (in UC). Correspondingly, there were 1885 significant PSI by location (listed in Table S1), 90 by ancestry, and none by disease or showing an interaction effect, implying that disease has a much smaller impact on splicing in each tissue than it does on overall expression.

The first principal component (PC1) of gene expression and the first two principal components (PC1 and PC2) of splicing provide particularly strong separation by location as seen in Fig. 1a,b, respectively, with the exception of samples from 8 individuals highlighted by the solid squares which are also extreme for PC2. We provide evidence in Fig. 1c,d and Fig S1 that these major

Table 1 Principal component variance analysis (PCVA) decomposition of sources of variance

\begin{tabular}{llll}
\hline & Gene expression & $\begin{array}{l}\text { PSI } \\
(\mathbf{1 0 8 , 0 9 1} \text { exon bins) }\end{array}$ & $\begin{array}{c}\text { PSI } \\
\text { (7001 exon bins) }\end{array}$ \\
\hline Principal component & & & $9.3 \%$ \\
PC1 & $28.9 \%$ & $7.6 \%$ & $6.9 \%$ \\
PC2 & $25.1 \%$ & $5.5 \%$ & $4.1 \%$ \\
PC3 & $5.2 \%$ & $4.1 \%$ & $3.2 \%$ \\
PC4 & $4.4 \%$ & $3.0 \%$ & $2.9 \%$ \\
PC5 & $3.5 \%$ & $2.6 \%$ & $2.7 \%$ \\
PC6 & $2.3 \%$ & $2.4 \%$ & $2.3 \%$ \\
PC7 & $2.0 \%$ & $2.3 \%$ & $2.0 \%$ \\
PC8 & $1.6 \%$ & $1.8 \%$ & $1.9 \%$ \\
PC9 & $1.6 \%$ & $1.7 \%$ & $1.6 \%$ \\
PC10 & $1.3 \%$ & $1.7 \%$ & $27.9 \%$ \\
SUM of PC1-PC10 & $75.9 \%$ & $33.0 \%$ & \\
Variance component & & & $5.3 \%$ \\
Ancestry & $2.3 \%$ & $5.8 \%$ & $0.5 \%$ \\
Location & $0.2 \%$ & $0.7 \%$ & $27.9 \%$ \\
Disease & $40.3 \%$ & $20.7 \%$ & $1.0 \%$ \\
Location*disease & $0.8 \%$ & $0.8 \%$ & \\
\hline
\end{tabular}

The table shows the percent variance in gene expression or splicing (PSI at two inclusion thresholds) explained by the first ten PCs and their sum, as well as the weighted contribution of each variance component term (ancestry, disease, location, and the interaction of disease and location) to these 10 PCs. Gene expression is for 18,929 genes, and number of PSI bins is before and after the final two stages of filtering ( $n=119$ samples for all) 


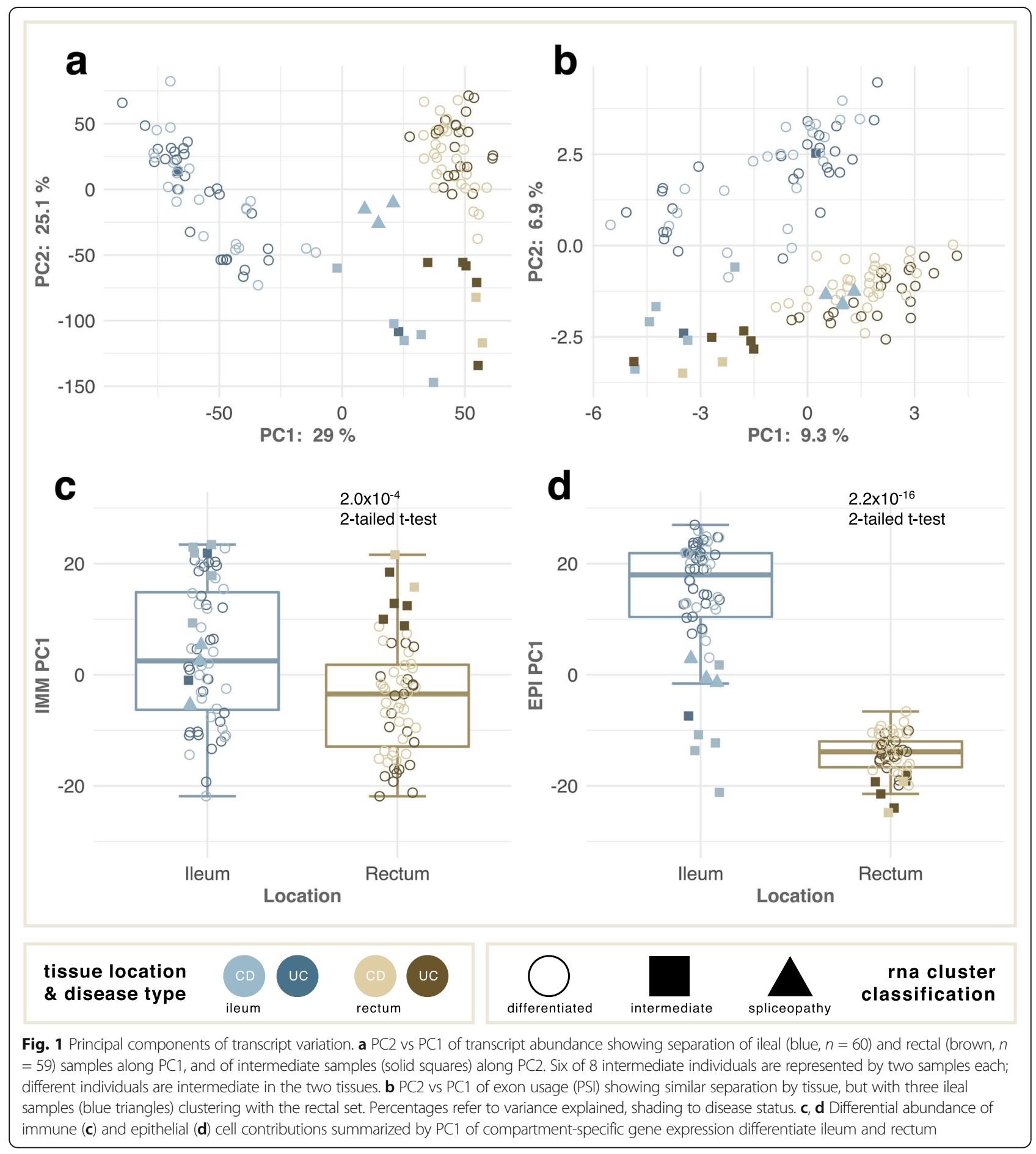

components of variation reflect the proportions of the three major tissue compartments [24, 25], specifically with elevated epithelial contribution to the ileum relative to the rectum and immune and fibroblast contributions to the intermediate samples. Analysis of the genes altered in the intermediate-type ileal and rectal cases indicate that the differentiation of these samples is likely driven by an amplified immune response. Of note, the
IBD-associated genes TLR2, TLR4, and NOD2 exhibit elevated expression in intermediate ileal and intermediate rectal samples.

\section{Aberrant profiles define "spliceopathies"}

Three ileal samples highlighted by the solid blue triangles in Fig. 1a,b (2 from one donor, 1 from another who did not have a paired ileal biopsy) have rectal-like 
splicing yet gene expression intermediate between the ileum and rectum (see also Fig S1). These two Crohn's disease cases thus have particularly altered splicing, suggesting that their disease is due to a "spliceopathy". Analysis of variance detected 284 differentially used splice sites in the three samples compared with the ileum (Table S2), and the heat map in Fig. 2a highlights how these ileal samples globally more resemble the rectum in terms of exon usage. These differentially used splice sites come from 246 genes, of which only 104 were found to be differentially expressed at the gene level, further supporting the suggestion of a "spliceopathy". A representative example, CEACAM1, itself an established IBD risk gene [26] whose product regulates mucosal inflammation via T-cells [27], is shown in Fig. 2b where exon bin 12 has low, rectal-like PSI in the ileum, whereas the other intermediate samples are more ileallike. The overall expression of the gene is normal (Fig. 2c).

There are two main isoform types of CEACAM1 that differ in the length of the cytoplasmic tail. The inhibitory functions of the long cytoplasmic tail isoform (CEAC $A M 1-L)$ are well studied, and CEACAM1- $L$ is known to be the predominantly expressed isoform in human lymphocytes. Though CEACAM1-S functions are less well characterized, it has been linked to mucosal immune regulation and recent studies show that intestinal $\mathrm{T}$ cells primarily express this isoform [28]. Exon bin 12 corresponds to exon 7 of the CEACAM1 gene, which is included only in the CEACAM1- $L$ isoform and also contains regions involved in the alternative splicing of this gene $[26,29]$. Analysis of this region did not identify any SNPs that may lead to the differential isoform ratio observed in these samples. While no other CEACAM family members exhibited altered splicing profiles, we did observe elevated gene expression of CEACAM5, known to be a marker of Crohn's disease, in the spliceopathy samples [30]. However, expression was consistent with the level seen among rectal samples, further supporting the hypothesis of a transcriptome-wide defect causing these ileal samples to resemble rectal tissue.

Underscoring that the defective splicing is transcriptome-wide, Fig. $2 \mathrm{~d}$ shows the fraction of exons in bins of differential usage for various contrasts, with the greatest deviations seen for the spliceopathy samples. Despite the widespread nature of the defective splicing, separation of samples by tissue type using PCA (principal component analysis) could also be performed reliably using just 96 of the 284 splice sites that were also differentially used in the rectal samples compared with the ileum, but did not distinguish the spliceopathy samples and rectal samples, making these exonic bins "rectallike". Gene ontology analysis of the genes encompassing these exons identified an enrichment of genes involved in fructose catabolism. Three out of five genes in the pathway (KHK, TKFC, and GLYCTK) had exons exhibiting rectal-like splicing in the ileal spliceopathy samples. In addition to differential isoform usage, overall transcript abundance of ketohexokinase (KHK) and triokinase $(T K F C)$ was also reduced in the spliceopathy samples, to a level intermediate between the rectum and ileum. Breath testing has been used to demonstrate that fructose malabsorption is quite common in individuals with ileal Crohn's disease [31], consistent with the hypothesis that an excess of short chain carbohydrates may be a trigger for pathogenesis.

Expansion of the gene ontology analysis to genes encompassing all 284 exons found to be differentially used in spliceopathy compared to ileum also identified enrichment of RNA splicing and spliceosome processes, suggesting that the rectal-like splicing observed in the spliceopathies is driven by an unknown aberration in the mRNA processing mechanisms of these patient's ileal tissue. However, there was no evidence from splicing, expression, or genotype data for the involvement of any of the three RNA-binding proteins known to influence alternative splicing of CEAC AM1 [29]. Two-way hierarchical clustering of PSI for the top 24 most spliceopathy-affected exons from 17 splicingrelated genes in Fig S2 shows that the two samples from one individual are clear outliers, while the single aberrant biopsy from the second individual falls within a small cluster of rectal-like ileal samples. These two cases thus likely have different genetic etiologies. It is not possible from this dataset to discern whether a single mutation is responsible for the profiles, or whether a combination of genetic and environmental factors lead to disruption of the splicing of these gene products, which then mediates the broader set of aberrant splice events.

\section{Discussion}

Our results establish that altered splicing is a relevant feature of the IBD gut. Since splicing is to some extent co-regulated with transcription [1,32], covariation of both aspects of gene expression is observed, for example in similarity of the principal components. An appreciable fraction of individuals have more rectal-like ileal expression and splicing also because of alterations in the proportions of epithelial, stromal, and immune cells. These differences are to some extent ancestry-biased, notably with elevated stromal (fibroblast) expression in Europeans relative to African Americans (Fig. 3.a,b). This observation extends our recent demonstration of ancestry-related differences in ileal gene expression involving pathways that also associate with disease severity [22]. Our ability to determine the cause and observe the downstream effects of the "spliceopathy" is limited by both the low number of individuals it was observed in and the design of our study. Future research could shed more light on the frequency, effects, and possible cause 


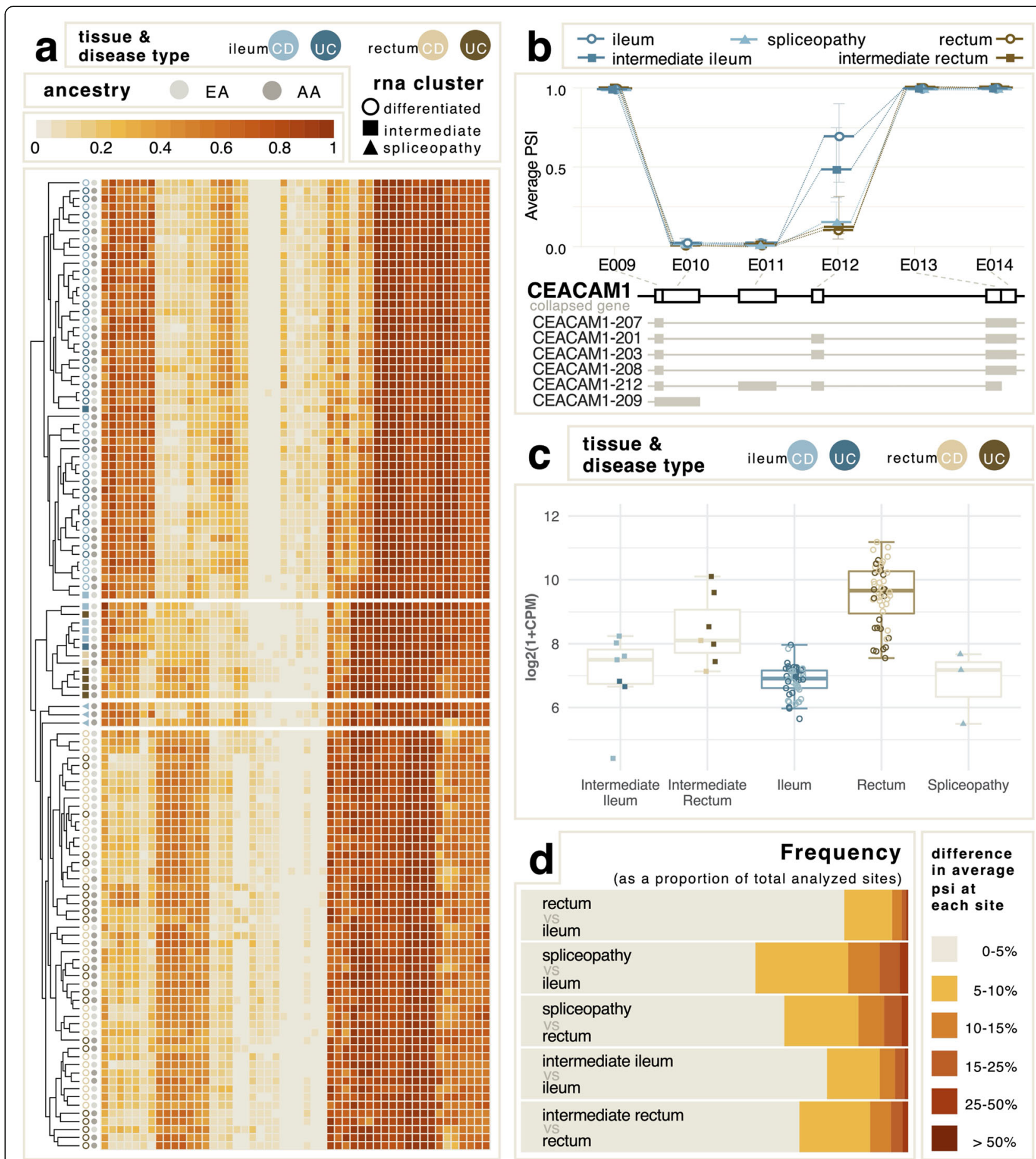

Fig. 2 Characteristics of the spliceopathy samples. a Heat map of the top 50 most differentially abundant exons showing broad clustering by tissue (rectum to the left) but not disease status. The intermediate samples cluster as a group, adjacent to the ileal spliceopathies which are closer to the rectal set and include aspects of both tissues. b Average PSI of exon bins 9 through 14 of CEACAM1, showing average levels of E012 (corresponding to CEACAM1 exon 7) differ by tissue and state. c Gene expression of CEACAM1 by cluster. Intermediate ileum and spliceopathy samples are not significantly different from differentiated ileum, whereas intermediate rectum and differentiated rectum are both significantly elevated relative to ileum. $\mathbf{d}$ The proportion of sites with indicated difference in average PSI for comparisons of ileum $(n=50)$ to rectum ( $n=52)$, spliceopathy ileum $(n=3)$ to both ileum and to rectum, and intermediate ileum $(n=7)$ or rectum $(n=7)$ to corresponding differentiated tissue. The most differential splicing is observed in each bin above $5 \%$ for the spliceopathies 


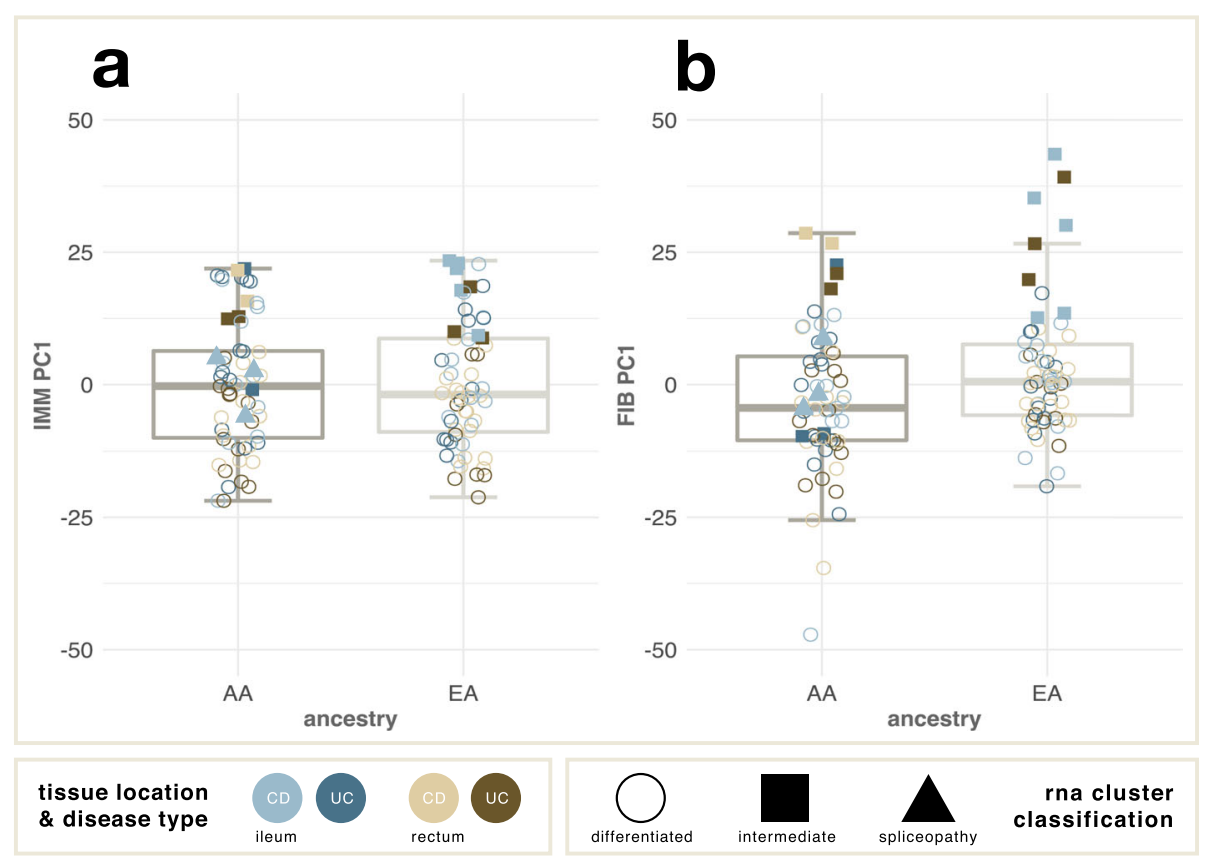

Fig. 3 Association of ancestry with tissue proportion in biopsies. a PC1 of immune-specific expression is similar between the two ancestry groups. b PC1 of fibroblast-specific expression is significantly different between the two ancestry groups, implying a reduced proportion of fibroblasts in the African ancestry biopsies ( $p=0.005,2$-tailed $t$ test). Note that the aberrant intermediate samples have particularly elevated fibroblast expression in both groups, whereas the two "spliceopathy" cases, both African American, have relatively normal fibroblast proportions

of this type of aberration by analyzing single-cell RNAseq and variants from whole genome sequencing in addition to bulk mRNA-seq in the rectal and ileal tissues. It will be important to define the molecular mechanisms responsible for the coordinated splicing defects and to evaluate whether they suggest personalized therapeutic interventions.

A further noteworthy aspect of this study is the development of a pipeline for quantitative analysis of splicing data from RNA-seq. The popular MISO (Mixture of Isoforms) protocol [33] incorporates fragment length distributions and exon-level abundance estimates into probabilistic estimation of altered isoform usage, but is intended for single sample comparisons. Several other existing approaches to detection of aberrant exon usage are incorporated into standard RNA-seq analysis tools [34-36], while an approach based on identification of novel or cryptic splice junctions in cases compared with controls, led to identification of the molecular basis for 17 of 48 previously undiagnosed neuromuscular disease cases [37]. Here we combine attributes of each of these algorithms along with quantitative evaluation of exon usage to identify suites of concurrent aberrant splicing in outlier individuals. PSI filtering (see Methods) allows for a focus on exons that are not constitutively expressed and therefore contribute to differential isoform usage, without being limited to annotated isoforms. Similar conclusions were observed at a variety of thresholds of inclusion, but careful filtering to rule out artefacts of low expression or exon coverage allowed us to focus on a core set of a few hundred genes perturbed in two cases of spliceopathy.

This study was performed using whole mRNA, which has long been the standard for gene expression analysis and, by extension, exon level and splice site analysis. However, several aspects of whole mRNA sequencing are not ideal for analysis of these smaller features, and a case can be made for targeted RNA-seq when at all possible. Our samples, following Illumina and ENCODE recommendations [38], had a median sequencing depth of 45 million reads. While this provides robust analysis at the gene level, it is quite limiting in terms of how many splice sites can be evaluated with accuracy. After removing exonic bins in genes with low or no coverage (60\% of sites), the mean number of informative reads per bin for any given sample is 169 , while the average maximum number of reads at a single site is over 750,000 . At over half of the remaining sites, the median PSI score across all samples is 0.99 , rendering those sites uninformative for differential usage analysis. A careful review of literature and public RNA-seq databases such as GTEX could identify genes that, though highly expressed in the target tissue, are not relevant for the proposed analysis. By using this information to create a targeted RNA-seq panel, researchers can achieve a higher read depth for a more robust analysis of splicing without needing to increase overall sequencing depth or sacrifice gene level analysis. 


\section{Conclusions}

Consistent with previous studies, we found tissue location to be the largest contributor to variability in gene expression and splicing. Though gene expression differences between tissues are often accompanied by changes in splicing, as one might expect since different cell types may utilize different isoforms, neither analysis shows the whole picture on its own. The observation of the ileal samples in two CD patients exhibiting intermediate gene expression but clear rectal-like splicing indicates that differential splicing is a previously unrecognized contributor to IBD disease pathology. Because the aberrations are seen in the full splicing profile rather than a specific aberrant splicing event, we refer to these cases as a "spliceopathy". Our results indicate that inclusion of splicing analysis when performing RNA-seq experiments for the study of human disease could play an important role in identifying additional contributions to the pathology of not only IBD, but also other complex diseases.

\section{Methods}

We analyzed whole mRNA sequencing profiles of 124 samples obtained from 34 young donors with IBD (age range 8-20 years). Duplicate biopsies of both the ileum and rectum were analyzed, in general, 4 samples per donor, although 6 donors were represented by only 3 samples and three by a single biopsy from each location. Individuals were closely matched for ancestry (18 European, 16 African American), sex (16 male, 18 female), disease type (20 Crohn's disease, CD; 14 ulcerative colitis, UC), and disease status at time of sampling (20 established cases, 14 cases at diagnosis). All donors were tumor free at biopsy. Following quality control, total transcript abundance was measured for 18,929 genes, and percent-spliced-in (PSI) estimates [39] were obtained for 7001 variable exon bins.

\section{Sequencing}

RNA was extracted, and library preparation was performed using the Illumina TruSeq Stranded mRNA kit. Paired-end 100bp stranded sequencing was performed for all samples on an Illumina HiSeq at a median read depth of 22.7 million (range: 10.2-106 million) read pairs.

\section{Preprocessing}

FastQC was run on raw fastq files to ensure mean phred scores per sequence and per base were above 27, to check consistency among samples in per sequence GC content, per base $\mathrm{N}$ content, sequence length distribution, and sequence duplication levels, and to check for the presence of adapters [40]. Samples were trimmed up to but not beyond the adapter using trimmomatic [41]. Samples were aligned with the STAR splice aware aligner to hg38 using the Gencode v29 primary assembly sequence and annotation [42, 43]. Default parameters were used with the following exceptions: to increase accuracy of splice site mapping and discovery, two-pass mode was invoked; novel splice junctions were required to have a minimum overhang of $8 \mathrm{bp}$, and a minimum of 5 unique reads was required for a splice site to be included in the splice junction output. To ensure each read used in downstream analysis was accurately mapped, and results were not affected by high homology regions such as pseudogenes, all multimapping reads (which map equally well to two locations in the genome) were filtered out. We further confirmed that all reads aligning to the CEACAM1 alternative splice bin did not align to the duplicate pseudogene [44], which possesses sufficiently divergent nucleotide sequence to prevent multi-mapping.

QC

In order to remove samples exhibiting extreme $5^{\prime}$ or $3^{\prime}$ bias or mapping issues that could affect splice calculations, sample quality was assessed using the Quality of RNA-Seq Tool-set (QoRTs) which evaluates cumulative gene diversity, gene-body coverage, and number of observed splice junction loci [45]. One rectal sample from individual 6 and one from individual 26 were observed to be extreme outliers in 3' bias and were removed, leaving both individuals with two ileal and one rectal samples. To confirm that each sample from an individual was indeed the same individual, variant calling was performed at Purcell's 5k sites following GATK best practices and identity-by-descent was compared using output from PLINK [46-48]. A PI_HAT minimum threshold of 0.7 was used to confirm a match between two samples. The single rectal sample from individual 18 failed the identity-by-descent QC measure, leading to the removal of all samples from individual 18 .

\section{Gene expression analysis}

Overall differential gene expression was performed with DESeq2, using the STAR raw read counts per gene output and including ancestry, disease, location, and the interaction of disease and location in the design formula [49]. Prior to analysis, genes were filtered for mean coverage $>5$ reads and both principal component analysis (PCA) and principal component variance analysis (PCVA) were performed on the final set of 18,929 genes, with results listed in Table 1 . PCA captures the major components of covariance of gene expression, and PVCA sums the amount of variance in each PC that is associated with the influencing factor, weighted by the variance in gene expression explained by the PC. We only analyzed the first 10PC of both RNA abundance and PSI since smaller PC explained less than $1 \%$ of the variance each and tend to capture differences among individuals or noise. 
Gene expression was also used to estimate abundance of specific cell types. Lists of genes expressed in immune, epithelial, and fibroblast cells were created from single-cell RNA-seq data for these cell types obtained from the colonic mucosa of ulcerative colitis patients [25], using thresholds of $>5$ counts per million (CPM) in one cell type and $<1$ CPM in the other two cell types. We then generated $\mathrm{PC} 1$ for each list and estimated the correlation with location and ancestry in order to evaluate the contribution of cell type abundance to these effects.

\section{Splicing analysis}

Splicing patterns between individuals were compared using the percent spliced in (PSI) metric, which was calculated per exonic bin for each sample. PSI is independent of library size and yields a score between 0 and 1 representing the proportion of isoforms that include a particular exonic bin. Inclusion (IR) and exclusion (ER) read counts were obtained following the protocol outlined in Schafer et al. [39] using the splice junction output from the STAR alignment, and the recommendation of requiring $>10$ ER to identify alternatively spliced exon bins was used to inform the following filtering steps. For each sample, if a site had $<10$ ER the PSI score was rounded up to the nearest tenth (IR $>10$ ) to lessen the impact of low exclusion counts or NA $(\mathrm{IR}<10)$ to indicate no coverage, allowing more exonic bins to be evaluated across all samples without low ER counts dominating the analysis. To limit analysis to genes expressed in both tissues, rows where one or more samples had no coverage were excluded (511,191 exon bins, leaving 108,091). Rows where all samples had the exact same PSI ( 0 or 1$)$ were removed $(64,129)$, reducing analysis to only those sites where one or more samples had variability in level of exon exclusion. Subsequently, to focus on splice bins with potential group-wise differences, further filtering was performed to exclude splice bins where $40 \%$ of samples were close to constitutively included or excluded (> $95 \%$ and $<5 \%$ PSI, respectively). This filtering reduced the original 619,282 potential splice sites to 7001 in the final analysis, with a mean PSI score of 0.45. PCA and PCVA were performed on the PSI estimates before and after the final stage of filtering, yielding very similar results presented in Table 1 . To identify differentially used exonic bins, linear mixed models were performed on the final set of PSI scores with the lme4 $\mathrm{R}$ package including fixed effects of disease, location, and ancestry and the interaction between disease and location, and a random effect of individual [50].

\section{Characterization of differential splicing in the spliceopathy samples}

A heatmap of 50 exon bins (Fig. 2a) was created to visualize differences in the splicing patterns of sample groups. Because the largest contributor of variance in
PSI was tissue location, the 50 most significant exonic bins by location obtained from the previously described analysis were used. Hierarchical clustering of samples for the 50 exonic bins was performed using the Euclidian distance and complete linkage method.

The difference between two PSI averages for each site was again used to observe the extent of variation between groups (Fig. 2d). Samples were split into ileum, rectum, intermediate ileum, intermediate rectum, and spliceopathy, and the difference in average PSI for each comparison was categorized at every exonic bin in the filtered 7001 exonic bins used for analysis.

For the identification of PSI sites in the spliceopathy samples that were significantly different from the differentiated ileum, the differentiated rectum, or both, exonic bin filtration criteria were relaxed slightly. To limit analysis to genes expressed in all three groups, rows where more than five ileal samples, five rectal samples, or one spliceopathy sample had no coverage were removed. The missing values in rows where 1-5 ileal or rectal samples had no coverage were replaced with the tissue location average at that site, allowing these additional 27,514 exonic bins to potentially be included in this analysis. The remainder of the filtration steps was carried out as before, this time reducing the original 619,282 potential splice sites to 9499 in the final analysis, with a mean PSI score of 0.43 . To identify differentially used exonic bins, linear mixed models were performed on the final set of PSI scores including fixed effects of group (ileum, rectum, or spliceopathy) and ancestry and a random effect of individual.

\section{Supplementary Information}

The online version contains supplementary material available at https://doi. org/10.1186/s40246-021-00347-y.

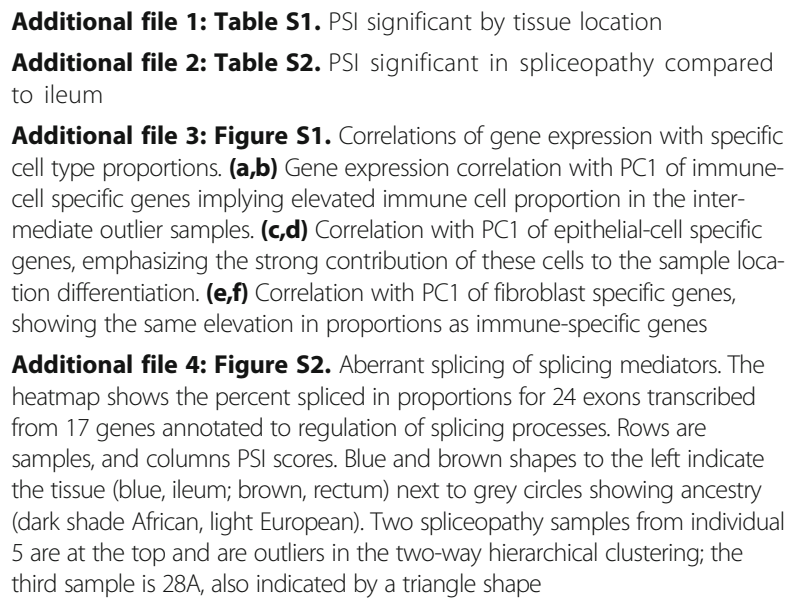

Additional file 3: Figure S1. Correlations of gene expression with specific cell type proportions. $(\mathbf{a}, \mathbf{b})$ Gene expression correlation with PC1 of immunecell specific genes implying elevated immune cell proportion in the intermediate outlier samples. (c,d) Correlation with PC1 of epithelial-cell specific genes, emphasizing the strong contribution of these cells to the sample location differentiation. $(\mathbf{e}, \mathbf{f})$ Correlation with PC1 of fibroblast specific genes, showing the same elevation in proportions as immune-specific genes

Additional file 4: Figure S2. Aberrant splicing of splicing mediators. The heatmap shows the percent spliced in proportions for 24 exons transcribed from 17 genes annotated to regulation of splicing processes. Rows are samples, and columns PSI scores. Blue and brown shapes to the left indicate the tissue (blue, ileum; brown, rectum) next to grey circles showing ancestry (dark shade African, light European). Two spliceopathy samples from individua 5 are at the top and are outliers in the two-way hierarchical clustering; the third sample is $28 \mathrm{~A}$, also indicated by a triangle shape

\section{Acknowledgements}

We are grateful to the study participants for agreeing to provide biopsy samples for this study and consenting to the research. 


\section{Authors' contributions}

Study concept and design: GG and HS; acquisition of data: JP and SK: statistical analysis and interpretation of data: $\mathrm{KB}$ and $\mathrm{HS}$; drafting and critical revision of manuscript: $\mathrm{KB}$ and $\mathrm{GG}$; obtained funding: $\mathrm{GG}$; administrative, technical, or material support: JP; study supervision: SK and GG. The author(s) read and approved the final manuscript.

\section{Funding}

Funding was provided by NIDDK grants 1-R01DK119991 to G.G., and 5R01DK087694 to S.K.

\section{Availability of data and materials}

The data discussed have been deposited in NCBI's Gene Expression Omnibus and are accessible through GEO Series accession number GSE158952. Relevant code can be accessed at the following Github repository: https:// github.com/kiera-gt/RectumlleumIBD-RNAseq.

\section{Declarations}

\section{Ethics approval and consent to participate}

Approval was obtained from the Institutional Review Boards of both the Georgia Institute of Technology and Emory University. The procedures used in this study adhere to the tenets of the Declaration of Helsinki. Informed consent was obtained from all individual participants included in the study.

\section{Consent for publication}

The authors affirm that human research participants provided informed consent for publication of their data.

\section{Competing interests}

The authors declare no competing interests.

\section{Author details}

'School of Biological Sciences and Center for Integrative Genomics, Georgia Institute of Technology, Atlanta, GA 30332, USA. ${ }^{2}$ Department of Pediatrics, Emory University, Atlanta, GA 30322, USA. ${ }^{3}$ Current address: insitro, San Francisco, CA 94080, USA.

Received: 15 April 2021 Accepted: 17 July 2021

Published online: 23 July 2021

\section{References}

1. Chabot B, Shkreta L. Defective control of pre-messenger RNA splicing in human disease. Journal of Cell Biology. 2016;212(1):13-27. https://doi.org/1 $0.1083 / j \mathrm{cb} .201510032$.

2. Bell SC, De Boeck K, Amaral MD. New pharmacological approaches for cystic fibrosis: promises, progress, pitfalls. Pharmacology \& therapeutics. 2015;145:19-34. https://doi.org/10.1016/.jpharmthera.2014.06.005.

3. Chen J, Weiss W. Alternative splicing in cancer: implications for biology and therapy. Oncogene. 2015;34(1):1-14. https://doi.org/10.1038/onc.2013.570.

4. Pistoni M, Ghigna C, Gabellini D. Alternative splicing and muscular dystrophy. RNA biology. 2010;7(4):441-52. https://doi.org/10.4161/rna. 7.4.12258

5. Vidak S, Foisner R. Molecular insights into the premature aging disease progeria. Histochemistry and cell biology. 2016;145(4):401-17. https://doi. org/10.1007/s00418-016-1411-1.

6. Xiong HY, Alipanahi B, Lee L, Bretschneider H, Merico D, Yuen RK, et al. The human splicing code reveals new insights into the genetic determinants of disease. Science. 2015:347:6218

7. Gelfman S, Wang Q, McSweeney KM, Ren Z, La Carpia F, Halvorsen M, et al. Annotating pathogenic non-coding variants in genic regions. Nature Communications. 2017:8(1):1-11.

8. Biamonti G, Bonomi S, Gallo S, Ghigna C. Making alternative splicing decisions during epithelial-to-mesenchymal transition (EMT). Cellular and Molecular Life Sciences. 2012;69(15):2515-26. https://doi.org/10.1007/s0001 8-012-0931-7.

9. Love JE, Hayden EJ, Rohn TT. Alternative splicing in Alzheimer's disease. J Parkinsons Dis Alzheimer Dis. 2015;2(2):6. https://doi.org/10.13188/2376-922 X.1000010.

10. Pellagatti A, Armstrong RN, Steeples V, Sharma E, Repapi E, Singh S, et al. Impact of spliceosome mutations on RNA splicing in myelodysplasia: dysregulated genes/pathways and clinical associations. Blood. 2018;132(12): 1225-40. https://doi.org/10.1182/blood-2018-04-843771.

11. Rabin SJ, Kim JMH, Baughn M, Libby RT, Kim YJ, Fan Y, et al. Sporadic ALS has compartment-specific aberrant exon splicing and altered cell-matrix adhesion biology. Human molecular genetics. 2010;19(2):313-28. https://doi. org/10.1093/hmg/ddp498.

12. Udd B, Krahe R. The myotonic dystrophies: molecular, clinical, and therapeutic challenges. The Lancet Neurology. 2012;11(10):891-905. https:// doi.org/10.1016/S1474-4422(12)70204-1.

13. Ng SC, Shi HY, Hamidi N, Underwood FE, Tang W, Benchimol El, et al. Worldwide incidence and prevalence of inflammatory bowel disease in the 21st century: a systematic review of population-based studies. The Lancet. 2017;390(10114):2769-78. https://doi.org/10.1016/S0140-6736(17)32448-0.

14. Furey TS, Sethupathy P, Sheikh SZ. Redefining the IBDs using genome-scale molecular phenotyping. Nature Reviews Gastroenterology \& Hepatology. 2019;16(5):296-311. https://doi.org/10.1038/s41575-019-0118-x.

15. Weiser M, Simon JM, Kochar B, Tovar A, Israel JW, Robinson A, et al. Molecular classification of Crohn's disease reveals two clinically relevant subtypes. Gut. 2018;67(1):36-42. https://doi.org/10.1136/gutjnl-2016-312518.

16. Digby-Bell JL, Atreya R, Monteleone G, Powell N. Interrogating host immunity to predict treatment response in inflammatory bowel disease. Nature Reviews Gastroenterology \& Hepatology. 2020;17(1):9-20. https://doi. org/10.1038/541575-019-0228-5.

17. Lee JC, Lyons PA, McKinney EF, Sowerby JM, Carr EJ, Bredin F, et al. Gene expression profiling of $\mathrm{CD} 8+\mathrm{T}$ cells predicts prognosis in patients with Crohn disease and ulcerative colitis. J Clin Invest. 2011;121(10):4170-9. https://doi.org/10.1172/JC159255.

18. Hyams JS, Thomas SD, Gotman N, Haberman Y, Karns R, Schirmer M, et al. Clinical and biological predictors of response to standardised paediatric colitis therapy (PROTECT): a multicentre inception cohort study. Lancet. 2019;393(10182):1708-20. https://doi.org/10.1016/S0140-6736(18)32592-3.

19. Kugathasan S, Denson LA, Walters TD, Kim M-O, Marigorta UM, Schirmer M, et al. Prediction of complicated disease course for children newly diagnosed with Crohn's disease: a multicentre inception cohort study. The Lancet. 2017; 389(10080):1710-8. https://doi.org/10.1016/S0140-6736(17)30317-3.

20. Marigorta UM, Denson LA, Hyams JS, Mondal K, Prince J, Walters TD, et al. Transcriptional risk scores link GWAS to eQTLs and predict complications in Crohn's disease. Nature genetics. 2017:49(10):1517-21. https://doi.org/10.103 8/ng.3936.

21. West NR, Hegazy AN, Owens BM, Bullers SJ, Linggi B, Buonocore S, et al. Oncostatin M drives intestinal inflammation and predicts response to tumor necrosis factor-neutralizing therapy in patients with inflammatory bowel disease. Nat Med. 2017;23(5):579-89. https://doi.org/10.1038/nm.4307.

22. Mo A, Krishnakumar C, Arafat D, Dhere T, Iskandar H, Dodd A, et al. African ancestry proportion influences ileal gene expression in inflammatory bowel disease. Cellular and molecular gastroenterology and hepatology. 2020; 10(1):203-5. https://doi.org/10.1016/j.jcmgh.2020.02.001.

23. Venkateswaran S, Marigorta UM, Denson LA, Hyams JS, Gibson G, Kugathasan S. Bowel location rather than disease subtype dominates transcriptomic heterogeneity in pediatric IBD. Cellular and molecular gastroenterology and hepatology. 2018;6(4):474-476.e3. https://doi.org/10.1 016/j.jcmgh.2018.07.001.

24. Mowat AM, Agace WW. Regional specialization within the intestinal immune system. Nature Reviews Immunology. 2014;14(10):667-85. https:// doi.org/10.1038/nri3738.

25. Smillie CS, Biton M, Ordovas-Montanes J, Sullivan KM, Burgin G, Graham DB, et al. Intra-and inter-cellular rewiring of the human colon during ulcerative colitis. Cell. 2019:178(3):714-30.e22.

26. Kelleher $M$, Singh $\mathrm{R}$, O'Driscoll $C M$, Melgar $S$. Carcinoembryonic antigen (CEAC AM) family members and inflammatory bowel disease. Cytokine \& growth factor reviews. 2019;47:21-31. https://doi.org/10.1016/j.cytogfr.2019.05.008.

27. Nagaishi $T$, Chen $Z$, Chen L, lijima $H$, Nakajima A, Blumberg R. CEACAM1 and the regulation of mucosal inflammation. Mucosal Immunol. 2008;1 (1): S39-42. https://doi.org/10.1038/mi.2008.50.

28. Chen L, Chen Z, Baker K, Halvorsen EM, da Cunha AP, Flak MB, et al. The short isoform of the CEACAM1 receptor in intestinal T cells regulates mucosal immunity and homeostasis via Tfh cell induction. Immunity. 2012; 37(5):930-46. https://doi.org/10.1016/j.immuni.2012.07.016.

29. Dery K, Gaur S, Gencheva M, Yen Y, Shively JE, Gaur RK. Mechanistic control of carcinoembryonic antigen-related cell adhesion molecule-1 (CEACAM1) splice isoforms by the heterogeneous nuclear ribonuclear proteins hnRNP L, 
hnRNP A1, and hnRNP M. Journal of Biological Chemistry. 2011;286(18): 16039-51. https://doi.org/10.1074/jbc.M110.204057.

30. Glas J, Seiderer J, Fries C, Tillack C, Pfennig S, Weidinger M, et al. CEACAM6 gene variants in inflammatory bowel disease. PLoS One. 2011;6(4):e19319. https://doi.org/10.1371/journal.pone.0019319.

31. Barrett JS, Irving P, Shepherd SJ, Muir JG, Gibson PR. Comparison of the prevalence of fructose and lactose malabsorption across chronic intestinal disorders. Alimentary Pharmacology Ther. 2009;30(2):165-74. https://doi. org/10.1111/j.1365-2036.2009.04018.x.

32. Neugebauer KM. Nascent RNA and the coordination of splicing with transcription. Cold Spring Harbor Perspect Biol. 2019;11(8):a032227. https:// doi.org/10.1101/cshperspect.a032227.

33. Katz Y, Wang ET, Airoldi EM, Burge CB. Analysis and design of RNA sequencing experiments for identifying isoform regulation. Nature methods. 2010;7(12):1009-15. https://doi.org/10.1038/nmeth.1528.

34. Hooper JE. A survey of software for genome-wide discovery of differential splicing in RNA-Seq data. Human Genomics. 2014;8(1):1-6.

35. Li Y, Rao X, Mattox WW, Amos Cl, Liu B. RNA-seq analysis of differential splice junction usage and intron retentions by DEXSeq. PloS one. 2015;10(9): e0136653. https://doi.org/10.1371/journal.pone.0136653.

36. Trapnell C, Hendrickson DG, Sauvageau M, Goff L, Rinn JL, Pachter L. Differential analysis of gene regulation at transcript resolution with RNA-seq Nature biotechnology. 2013;31(1):46-53. https://doi.org/10.1038/nbt.2450.

37. Cummings BB, Marshall JL, Tukiainen T, Lek M, Donkervoort S, Foley AR, et al. Improving genetic diagnosis in Mendelian disease with transcriptome sequencing. Science Transl Med. 2017;9:386.

38. Illumina Technical Support. Considerations for RNA-Seq read length and coverage [Internet]. 2017 [updated 2020 Nov 11]. Available from: https:// support.illumina.com/bulletins/2017/04/considerations-for-rna-seq-readlength-and-coverage-.html.

39. Schafer S, Miao K, Benson CC, Heinig M, Cook SA, Hubner N. Alternative splicing signatures in RNA-seq data: percent spliced in (PSI). Curr Protoc Human Genet. 2015;87(1):11.16.1-11.16.14. https://doi.org/10.1002/0471142 905.hg1116s87.

40. Andrews S. FastQC: a quality control tool for high throughput sequence data [Online]. 2010. Available from: http://www.bioinformatics.babraham.ac. uk/projects/fastqc

41. Bolger AM, Lohse $M$, Usadel B. Trimmomatic: a flexible trimmer for Illumina sequence data. Bioinformatics. 2014;30(15):2114-20. https://doi.org/10.1093/ bioinformatics/btu170.

42. Dobin A, Davis CA, Schlesinger F, Drenkow J, Zaleski C, Jha S, et al. STAR ultrafast universal RNA-seq aligner. Bioinformatics. 2013;29(1):15-21. https:// doi.org/10.1093/bioinformatics/bts635.

43. Frankish A, Diekhans M, Ferreira A-M, Johnson R, Jungreis I, Loveland J, et al. GENCODE reference annotation for the human and mouse genomes. Nucleic Acids Res. 2019:47(D1):D766-D73. https://doi.org/10.1093/nar/gky955.

44. Beauchemin N, Draber P, Dveksler G, Gold P, Gray-Owen S, Grunert F, et al. Redefined nomenclature for members of the carcinoembryonic antigen family. Experimental cell research. 1999;252(2):243-9. https://doi.org/10.1 006/excr.1999.4610.

45. Hartley SW, Mullikin JC. QoRTs: a comprehensive toolset for quality control and data processing of RNA-Seq experiments. BMC Bioinformatics. 2015;16(1):1-7.

46. Purcell S, Neale B, Todd-Brown K, Thomas L, Ferreira MA, Bender D, et al. PLINK: a tool set for whole-genome association and population-based linkage analyses. Am J Human Genet. 2007;81(3):559-75. https://doi.org/10.1086/519795.

47. Purcell SM, Moran JL, Fromer M, Ruderfer D, Solovieff N, Roussos P, et al. A polygenic burden of rare disruptive mutations in schizophrenia. Nature. 2014;506(7487):185-90. https://doi.org/10.1038/nature12975.

48. Van der Auwera GA, Carneiro MO, Hartl C, Poplin R, Del Angel G, LevyMoonshine A, et al. From FastQ data to high-confidence variant calls: the genome analysis toolkit best practices pipeline. Curr Protocols Bioinformatics. 2013;43(1):11.10.1-11.10.33.

49. Love Ml, Huber W, Anders S. Moderated estimation of fold change and dispersion for RNA-seq data with DESeq2. Genome biology. 2014;15(12):550. https://doi.org/10.1186/s13059-014-0550-8.

50. Bates $\mathrm{D}$, Mächler M, Bolker B, Walker S. Fitting linear mixed-effects models using Ime4. J Statistical Software. 2015;67(1):48.

\section{Publisher's Note}

Springer Nature remains neutral with regard to jurisdictional claims in published maps and institutional affiliations.

\section{Ready to submit your research? Choose BMC and benefit from}

- fast, convenient online submission

- thorough peer review by experienced researchers in your field

- rapid publication on acceptance

- support for research data, including large and complex data types

- gold Open Access which fosters wider collaboration and increased citations

- maximum visibility for your research: over $100 \mathrm{M}$ website views per year

At BMC, research is always in progress.

Learn more biomedcentral.com/submissions 